\title{
Simulink toolbox for simulation and analysis of optical fiber links
}

\section{Claudio de Melo, Cesar Lima, Licinius de Alcantara, Ronaldo dos Santos, Joao Costa}

Claudio F. de Melo Jr., Cesar Albuquerque Lima, Licinius D.S. de Alcantara, Ronaldo O. dos Santos, Joao Chrisustomo Weyl Albuquerque Costa, "Simulink toolbox for simulation and analysis of optical fiber links," Proc. SPIE 3831, Sixth International Conference on Education and Training in Optics and Photonics, (16 June 2000); doi: 10.1117/12.388731

Event: Education and Training in Optics and Photonics (ETOP'99), 1999, Cancun, Mexico 


\title{
A Simulink ${ }^{\mathrm{TM}}$ toolbox for simulation and analysis of optical fiber links
}

\author{
Cláudio F. de Melo JR, César A. Lima, Licinius D. S. de Alcantara, Ronaldo O. dos Santos and \\ João C. W. A. Costa. \\ Laboratório de Eletromagnetismo Aplicado - Departamento de Engenharia Elétrica \\ Centro Tecnológico - Universidade Federal do Pará
}

\begin{abstract}
This paper presents a complete toolbox to be used together with the MATLAB / SIMULINK software. The versatility of this software is used to form analysis blocks in the time and frequency domains, allowing the user to simulate optical fiber links of any topology, as long as the analysis of its performance face changes in the values of its parameters. Results for LED (F.D.), Quantum Well (T.D.) and Fabry Perot (F. D.) LASERs are shown.
\end{abstract}

KeyWords: Optical Links, Simulation of optical links, LED, Fabry-Perot LASER, Quantum Well LASER, Rate Equations, Simulink ${ }^{\mathrm{TM}}$.

\section{INTRODUCTION}

The use of educational softwares is widely recommended in present days as a complementary tool in electrical engineering and associated disciplines. This results primarily from the fact that these softwares offer convenient means for mathematical manipulations and for the visualization of the physical phenomena associated with these disciplines. In addition, the high cost of modern laboratories in this area enhances the interest of a more diversified use of these softwares [1].

The Matlab, Mathcad, Maple and Mathematica softwares, for example, are widely used in engineering, not only as a teaching but also as a professional tool. Besides, these softwares provide a friendly computational environment in which the facilities of numerical and/or classical algebraic equations solutions combine with the facilities of visualization and document integration. As a result, the difficulty, quite often encountered by undergraduate and graduate students, is partially overcome from the moment that these students are faced with a didactic computational tool and of easy comprehension, implemented in an environment familiar to the students.

The analysis need of the performance of each component individually or in group in a optical fiber telecommunication link has given reason for the creation of a toolbox, which should be used along with the Matlab/Simulink software, allowing the programmer to concentrate only in the validity of the model being used.

The toolbox may be understood as a group of blocks which represent the various components of a optical link, such as light sources (LEDs and lasers), optical fibers (multimode and monomode), photodetectors (PIN or APD) and allow the simulation of its dynamic behavior individually or in group. Its main characteristic is therefore its flexibility, for there is no topology defined, and the user is the one who decides the way the system is built up.

\section{DEVICE MODELS}

In this section is shown how to construct Simulink blocks using the mathematical (physical) model. The devices can be analyzed using T.D. or F.D. model. For illustrating proposals, the source models for LED, Fabry-Perot Laser and MQW laser will be described. In the F.D. the Fourier Transform (F.T.) of the optical power, $P_{e}(f)$ (watts) can be expressed as:

$$
P_{e}(f)=H_{T}(f) \cdot I_{d}(f),
$$

Where $H_{T}(f)$ is the transfer function of the source and $I_{d}(f)$ is the F. T. of the injected current (A). The transfer function can be decomposed in two parts:

$$
H_{T}(f)=H_{T}(0) \cdot H_{T}^{*}(f)
$$

Correspondence: e-mail : jweyl@ufpa.br; P.O Box: 8619, ZIP 66075-900, Belém-Pa-Brazil

Simulink ${ }^{T M}$ is a trademark of The Mathworks Inc. 
In the previous equation, $H_{T}(0)$ is the quantum efficiency of the light source (W/A), $H_{T}^{*}(f)$ is the normalized frequency response of the light source.

\subsection{LED}

For the LEDs the transfer function can be expressed as [4]

$$
H_{T}(0)=\left(\frac{h . c}{\lambda . q}\right) \eta_{\text {int }} \eta_{i n j} \eta_{e x t}
$$

where :

$$
\begin{gathered}
\eta_{\text {int }}=\frac{\tau_{n r}}{\tau_{n r+} \tau_{r}} \\
\eta_{\text {ext }}=\left[1-\left(\frac{n_{s}-n_{m}}{n_{s}+n_{m}}\right)^{2}\right]\left[1-\cos \left(\frac{n_{m}}{n_{s}}\right)\right]
\end{gathered}
$$

The term $H_{T}^{*}(f)$ in (2) is expressed by

where:

$$
H_{T}^{*}(f)=\frac{1}{1+j f / f_{c}}
$$

$$
f_{c}=\frac{1}{2 \pi \tau_{r}}
$$

The meaning of parameters in (3)-(7) are given at TABLE I.

\subsection{Multimode Fabry-Perot:}

For the Fabry-Perot laser the transfer function can be expressed as [4]

$$
H_{T}(0)=\left(\frac{h . c}{\lambda . q}\right) \eta_{\text {int }} \eta_{e x t}\left[\frac{I_{d}-I_{t h}}{I_{d}}\right]
$$

TABLE I

PARAMETERS OF (3),(7)

\begin{tabular}{|c|c|c|c|}
\hline$\eta_{e x t}$ & External Quantum efficiency & $Q$ & Electron charge $\left(1.60218 \times 10^{-19} \mathrm{C}\right)$ \\
\hline$\eta_{\text {int }}$ & Internal Quantum efficiency & $\tau_{n r}$ & Nonradiative recombination lifetime \\
\hline$\eta_{i n j}$ & Injection current efficiency & $\tau_{r}$ & Radiative recombination lifetime \\
\hline$h$ & Planck's constant $\left(6.62 \times 10^{-34} j . s\right)$ & $n_{s}$ & Semiconductor refraction index \\
\hline$c$ & Light velocity in the vacuum $\left(2.99793 \times 10^{8} \mathrm{~m} / \mathrm{s}\right)$ & $n_{m}$ & Refraction index (air =1) \\
\hline$\lambda$ & Emission wavelength $(\mathrm{m})$ & $f_{c}$ & Optical cutoff frequency (3 dB) \\
\hline
\end{tabular}


Where:

$$
\eta_{\text {ext }}=\frac{\ln \left(\frac{1}{R_{1}}\right)}{\gamma \cdot l+\ln \left(\frac{1}{R_{1}}\right)}
$$

and $\eta_{\text {int }}$ is the same as in (4).

where:

$$
H_{T}^{*}(f)=\frac{f_{0}^{2}}{f_{0}^{2}-4 \pi^{2} f^{2}+j \beta 2 \pi f}
$$

$$
\begin{aligned}
f_{0}^{2} & =\frac{\left(I_{0}-I_{t h}\right)}{\tau_{s p} \tau_{p h} I_{t h}}, \\
\beta & =\frac{I_{0}}{\tau_{s p} I_{t h}} .
\end{aligned}
$$

The description of new parameters at (8)-(12) are given in TABLE II.

TABLE II

PARAMETERS OF (8)-(12)

\begin{tabular}{|c|c|c|c|}
\hline$I_{d}$ & Injected current & $I_{0}$ & Polarization current (A) \\
\hline$I_{t h}$ & The threshold current (A). & $\tau_{s p}$ & Carrier recombination lifetime (s) \\
\hline$R_{1}$ & Mirror reflectancy (m) & $\beta$ & Dumping frequency (Hz) \\
\hline$\gamma$ & loss coeficient & $f_{0}$ & Resonant frequency (Hz). \\
\hline$l$ & Cavity longitudinal dimension (m) & & \\
\hline
\end{tabular}

Obs : the former expressions are valid only for $I_{d}>I_{t h}$, i.e., in the stimulated emission region

\subsection{Quantum-Well (QW) LASER.}

To obtain the response of the quantum-well (QW) LASER was not used a model, but a implementation in the time domain through a block diagram using SIMULINK, according to the following rate equations [2]:

$$
\begin{gathered}
\frac{d N}{d t}=\frac{I}{q V_{a c t}}-g_{0}\left(N-N_{0}\right)(1-\varepsilon S) S-\frac{N}{\tau_{n}}+\frac{N_{e}}{\tau_{n}} \\
\frac{d S}{d t}=\Gamma g_{0}\left(N-N_{0}\right)(1-\varepsilon S) S+\frac{\Gamma \beta N}{\tau_{n}}-\frac{S}{\tau_{p}} \\
\frac{S}{P_{f}}=\frac{\Gamma \tau_{p} \lambda_{0}}{V_{a c t} \eta h c}=v
\end{gathered}
$$

The terms of the above equation are described in TABLE III 
TABLE III

PARAMETERS OF (13)-(15)

\begin{tabular}{|l|l|l|l|}
\hline$N$ & Active region carrier density & $\beta$ & Spontaneous emission coupling factor \\
\hline$S$ & Photon density & $\tau_{p}$ & Photon lifetime \\
\hline$P_{f}$ & LASER output power & $\eta$ & Differential quantum efficiency per facet \\
\hline$I$ & Injection current & $\lambda_{0}$ & Lasing wavelength \\
\hline $\mathrm{V}_{\text {act }}$ & Active region volume & $Q$ & Electron charge \\
\hline$g_{0}$ & Gain coefficient. & $H$ & Planck's constant \\
\hline$N_{0}$ & Optical transparency density. & $C$ & Light velocity (vacuum) \\
\hline $\mathcal{E}$ & Fenomenological gain-saturation term & $N_{e}$ & Equilibrium carrier density \\
\hline$\tau_{n}$ & Carrier lifetime & $\Gamma$ & Optical confinement factor \\
\hline
\end{tabular}

Accordingly with [1], the standard rate equations that use a linear gain-saturation term of the form $(1-\varepsilon) S$ can possess three dc solution regimes for nonnegative values of injection current, which two of them are nonphysical solutions, characterized for negative power and high power solutions. For the parameter values used in this paper, the nonphysical solutions would happen above a injection current value between 0.5 and $2 \mathrm{~A}$ [1], which is higher than typical values applied in these simulations

\section{TOOLBOX PRESENTATION}

The developed blocks are separated in five main groups for the convenience of the user. They are: Signal Origin, Light Sources (explained here), Optical Fibers, Photodetectors and Passive Devices. Anytime one of these groups are "doubleclicked" with the mouse, the user gets access to the windows containing blocks representing each component of the system. These windows are shown in Figures 1 through 6 Using this blocks, it is possible to assemble a simulation diagram, as will be demonstrated in the next section.

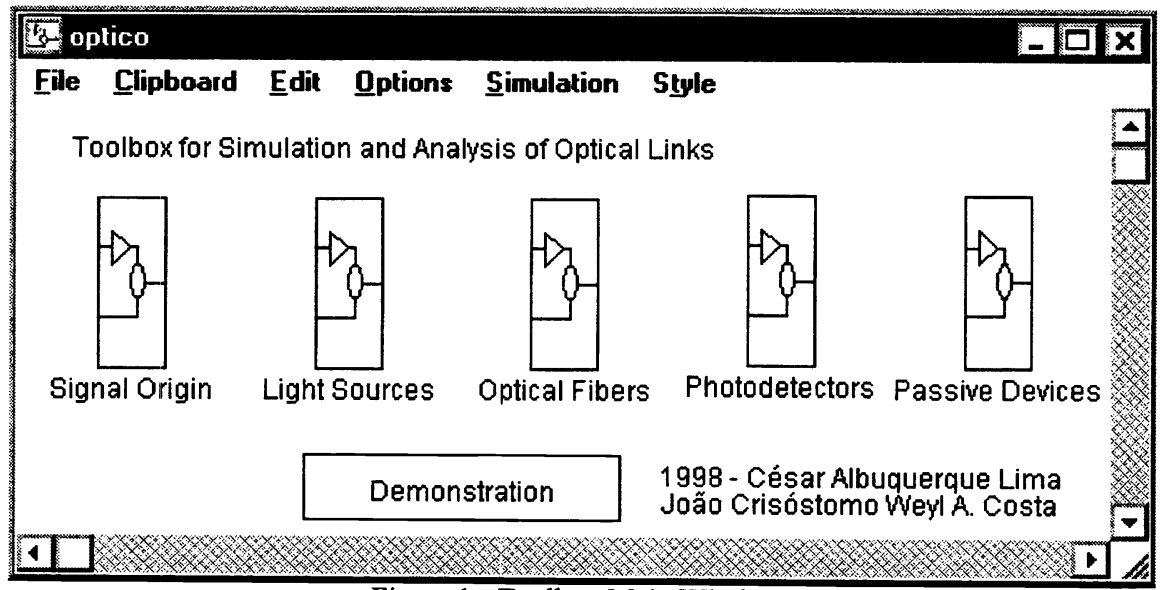

Figure 1 - Toolbox Main Window. 


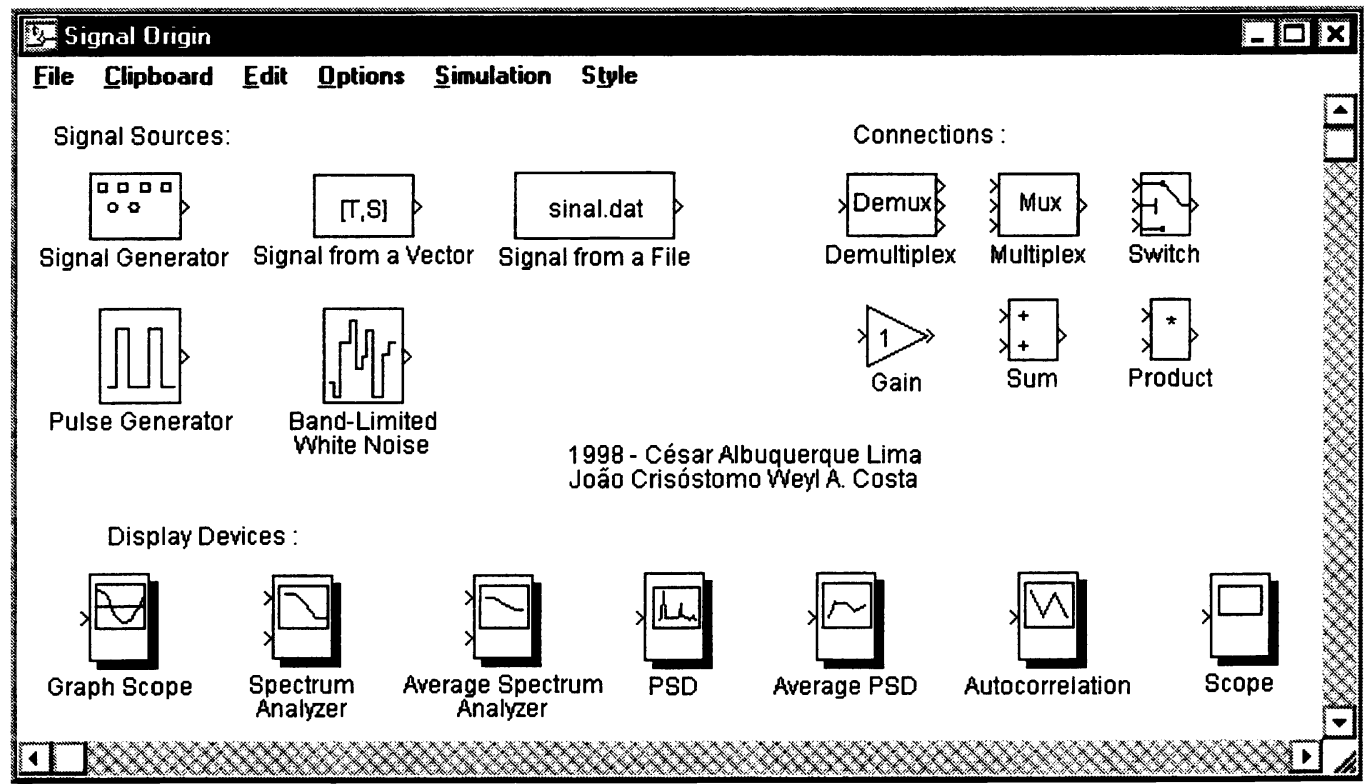

Figure 2 - Signal Origin Group Window.

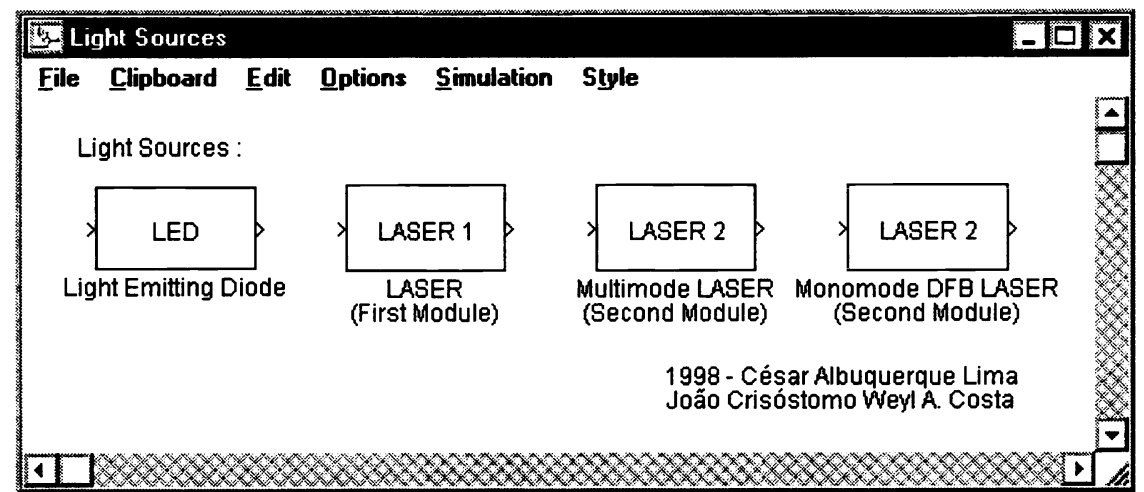

Figure 3 - Light Sources Group Window.

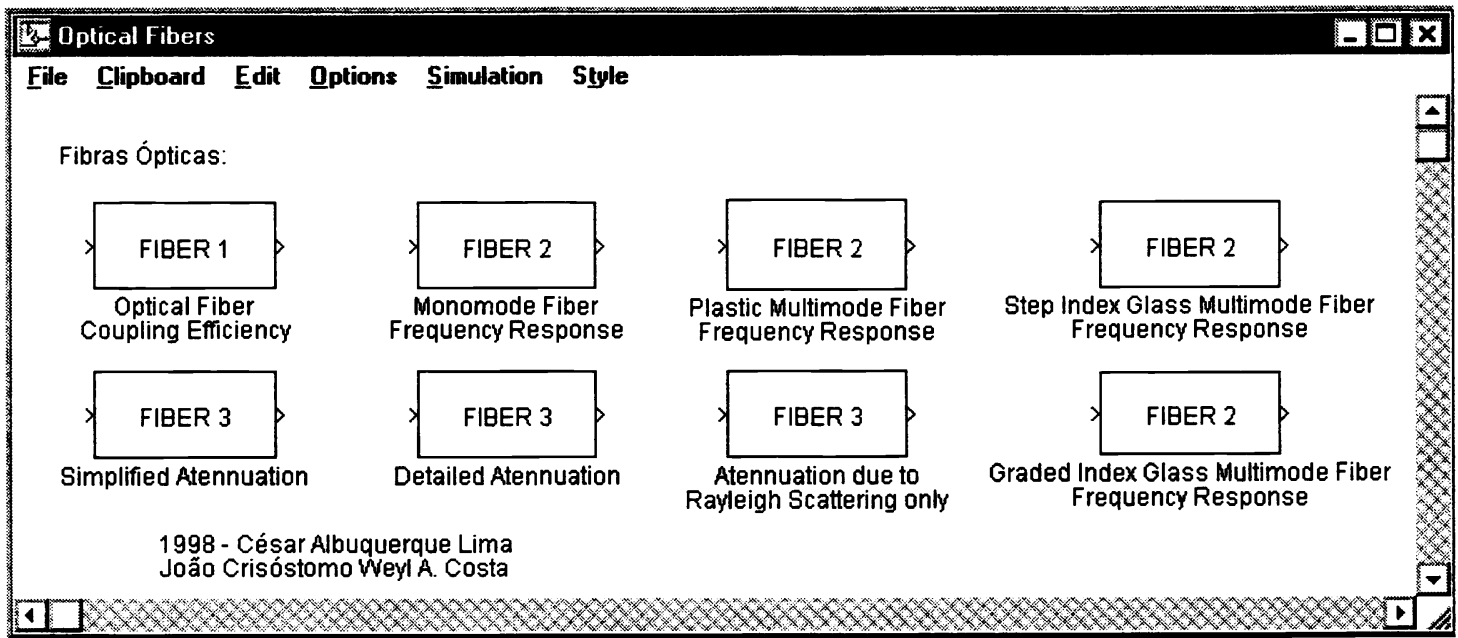

Figure 4 - Optical Fibers Group Window 


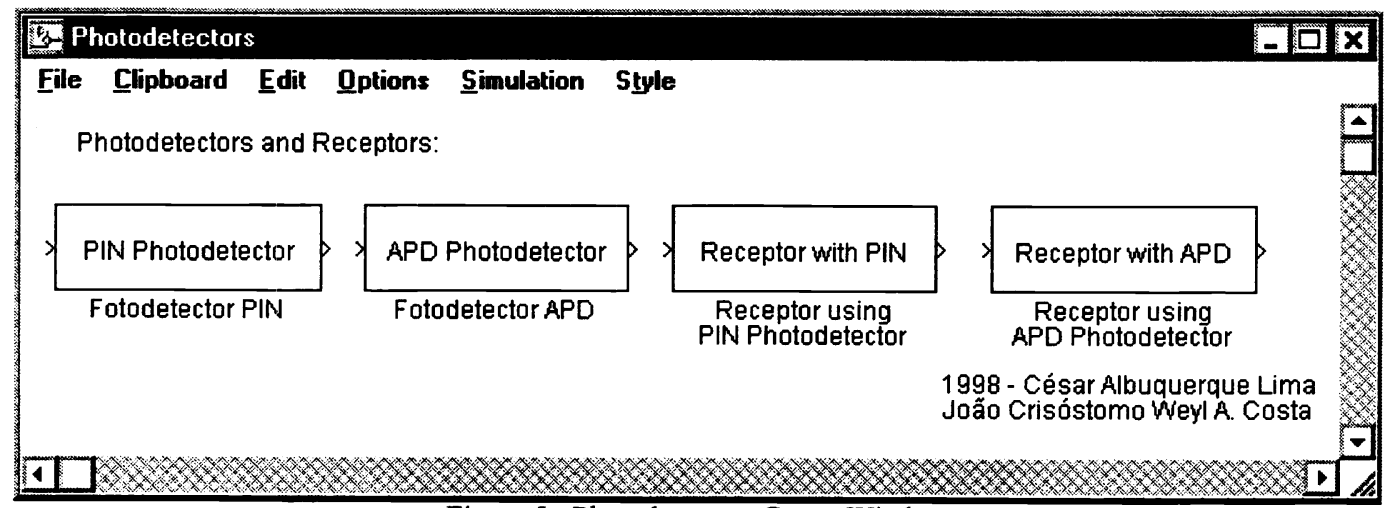

Figure 5 - Photodetectors Group Window

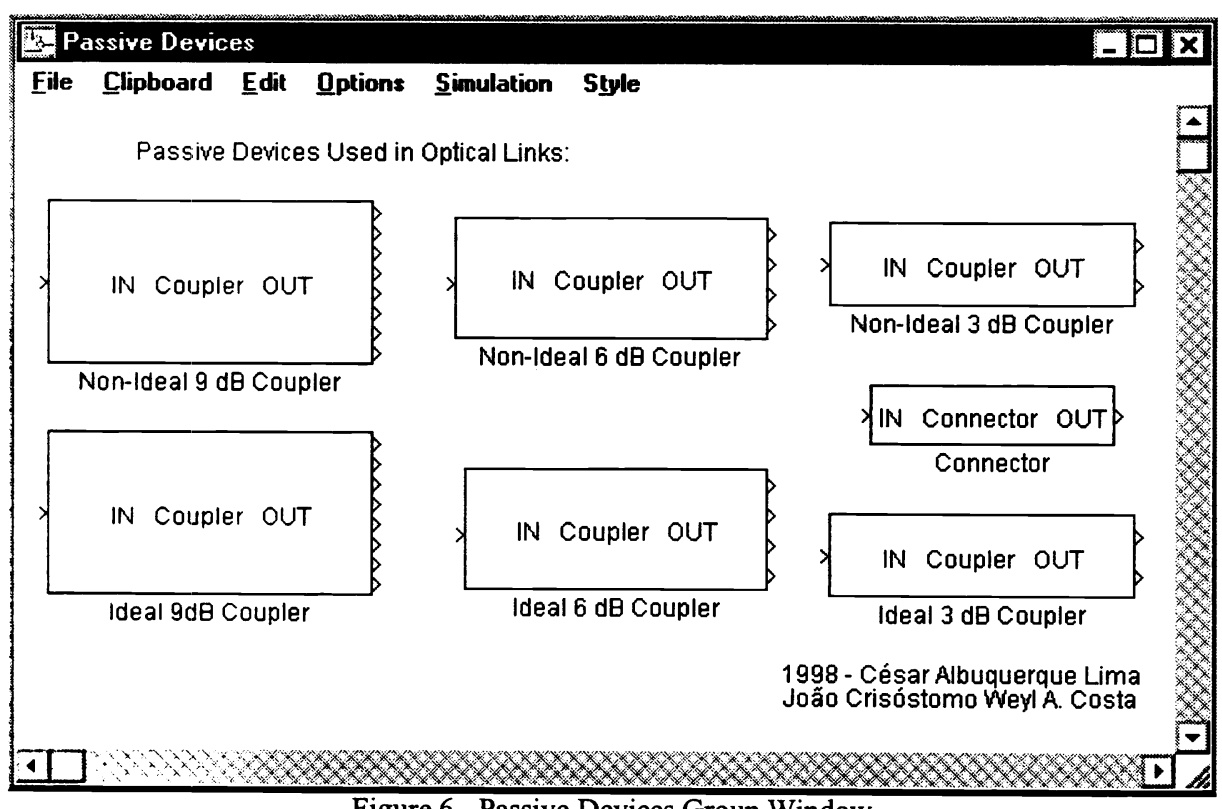

Figure 6 - Passive Devices Group Window

\subsection{LED}

In fig.7 can be seen the LED block, with other blocks like signal generator and scope. The user can quickly change th parameters of a LED block, as shown in fig.8.

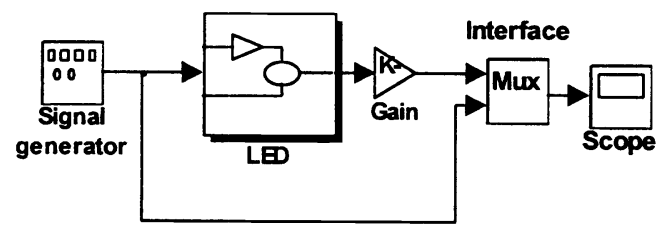

Fig. 7- Structure used to simulate the behavior of a LED

Values used in the simulations of this work are also shown in figs. 8, 11 and 14. Using this structure to simulate the behavior of a LED, and plotting the optical power emitted when the input is a test square wave, can be seen in the fig. 9. 


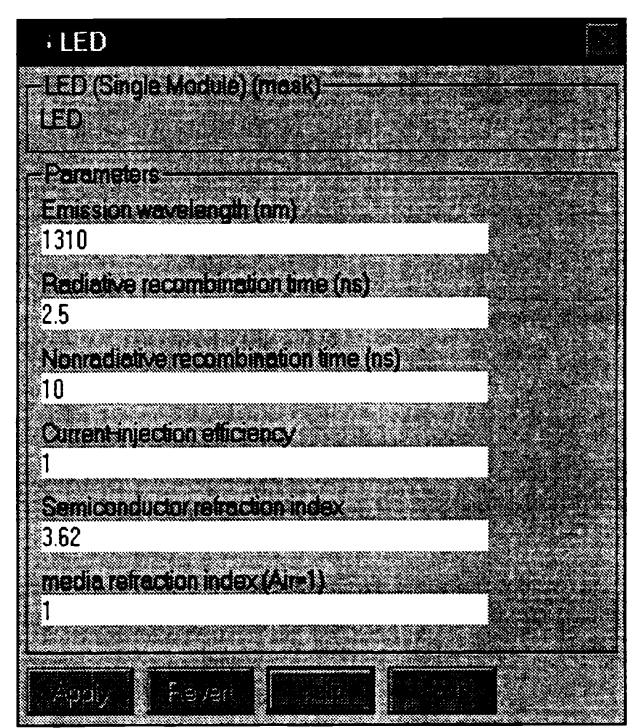

Fig.8 - Dialog box to the LED parameters.

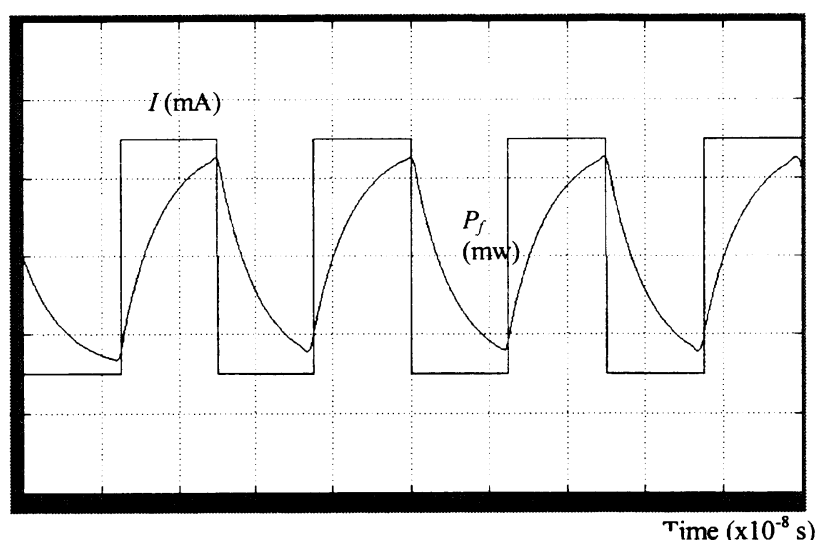

Fig.9 - Simulation Results to the LED.

\subsection{Fabry-Perot LASER (multimode)}

The block diagram to the simulation of the LASER diode is shown below. This is a model of multimode Fabry-Perot LASER.

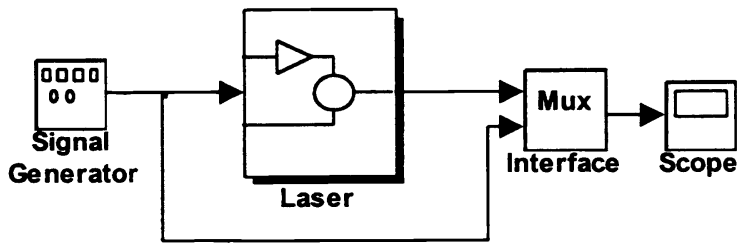

Fig.10- Structure used to simulate a LASER diode.

Activating the LASER block, the following Dialog box will open, allowing the user to adjust the parameters seen in the fig. 11. 


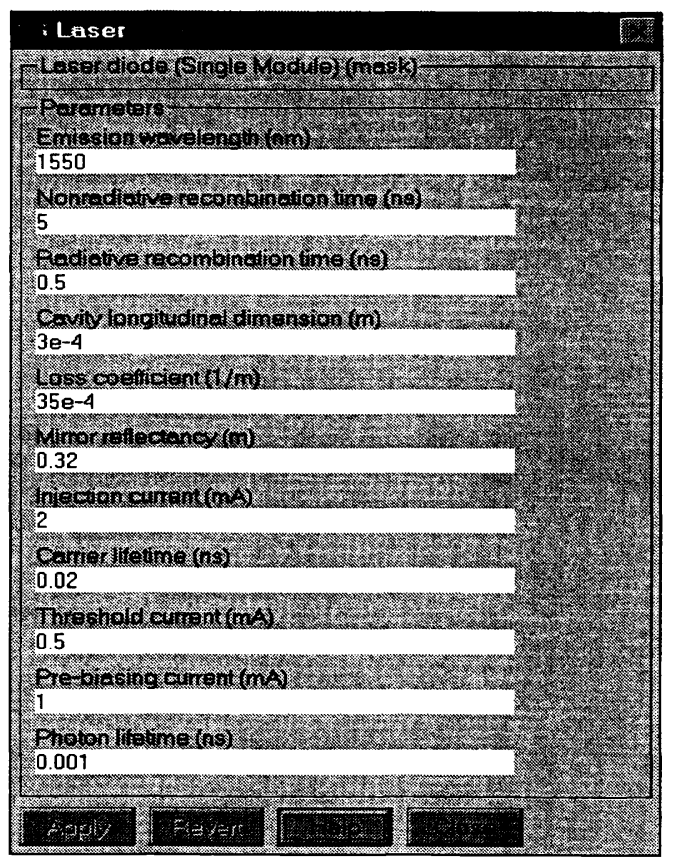

Fig.11- Dialog box with LASER diode parameters.

The results of simulation (fig. 12), show the behavior of a LASER diode, with a square wave current of input signal.

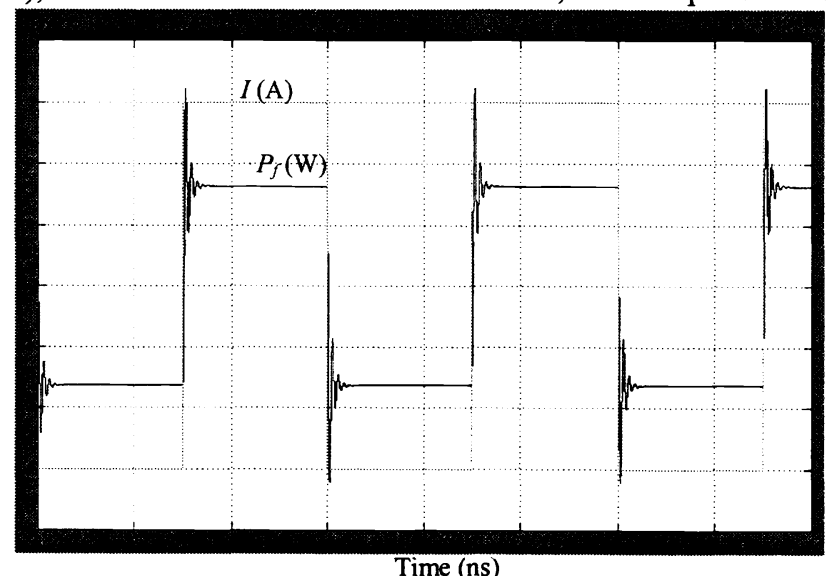

Fig.12- Simulation Results to the LASER Diode

Note: To permit the simulation of the Fabry-Perot LASER, as well as the QW LASER, to be discussed in the following session, was necessary to adjust the time step in "simulations parameters" dialog box, to fix (Runge-Kutta method), using a time step lower than the default, to guarantee the stability of digital simulation. 


\subsection{Quantum-Well LASER}

The block diagram related to the simulation of the LASER diode is shown in the fig. 13.Fig. 14 illustrates the dialog box for input parameters, activated by double clicking the Rate equation block, allowing the simulation of QW LASER for different materials and structures. Accordingly with (13)-(15), the "rate equations" block in fig 13 is composed by the structure shown in fig.15. Fig.16 shows the plot of optical power output corresponding to the values given in fig.15 (default values), where the injected current varies between 0 and $10 \mathrm{~mA}$ with a period of $40 \mathrm{nS}(25 \mathrm{Mhz})$. It Can be observed in this figure that there is a significant delay in the response, considering that the lower level of the injected current is below the threshold current to cause lasing. To other experienced simulations, varying the injected current between 8 and $10.5 \mathrm{~mA}$, the output optical power follows the shape of the input signal in a better manner, with less delay, with a consequence that a greater operating frequency could be used. However, the rising of the superior level of the injected current, the overshoot becomes bigger, indicating a reduction in the relative stability of the system. Fig.17 shows the relation between the photons density S, carrier density $\mathrm{N}$ and the optical output power to the same injection current used in fig. 16.

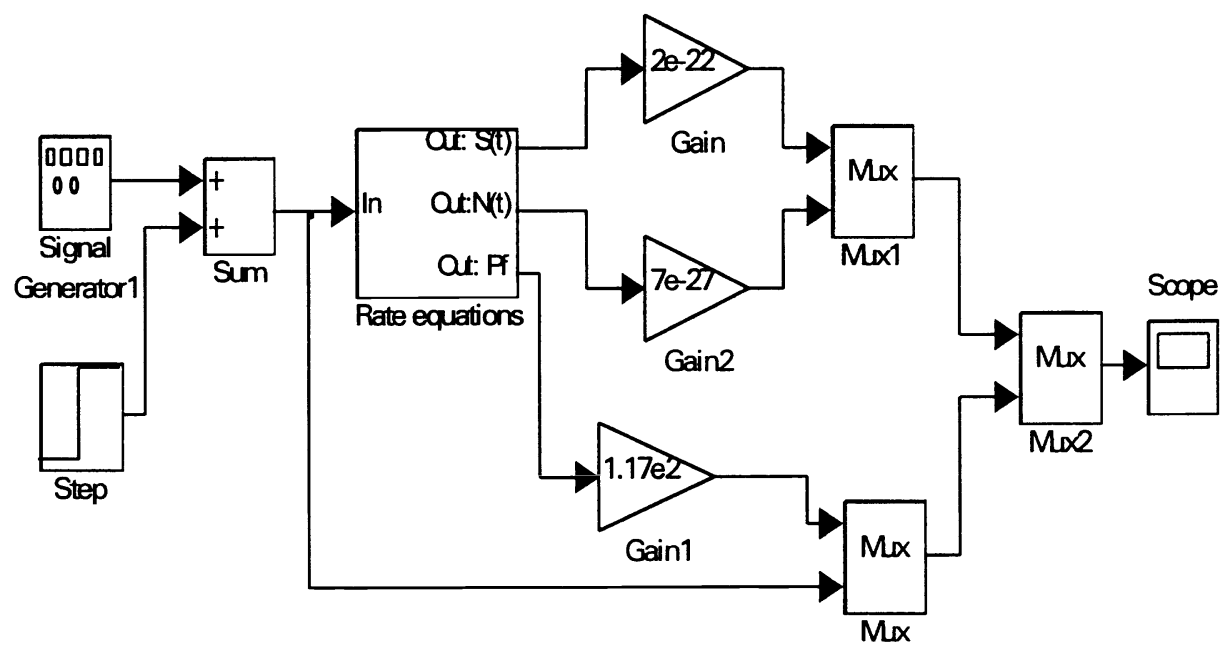

Fig.13 - Block diagram used to simulate a QW LASER

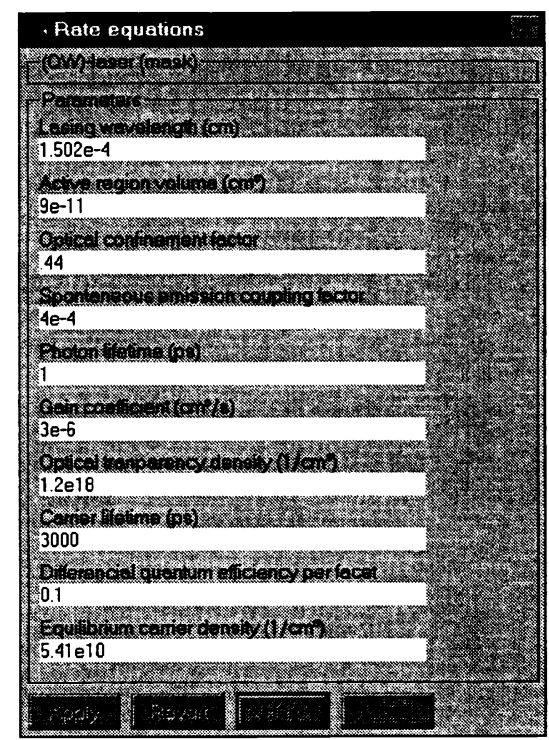

Fig.14- Dialog box for the QW LASER 


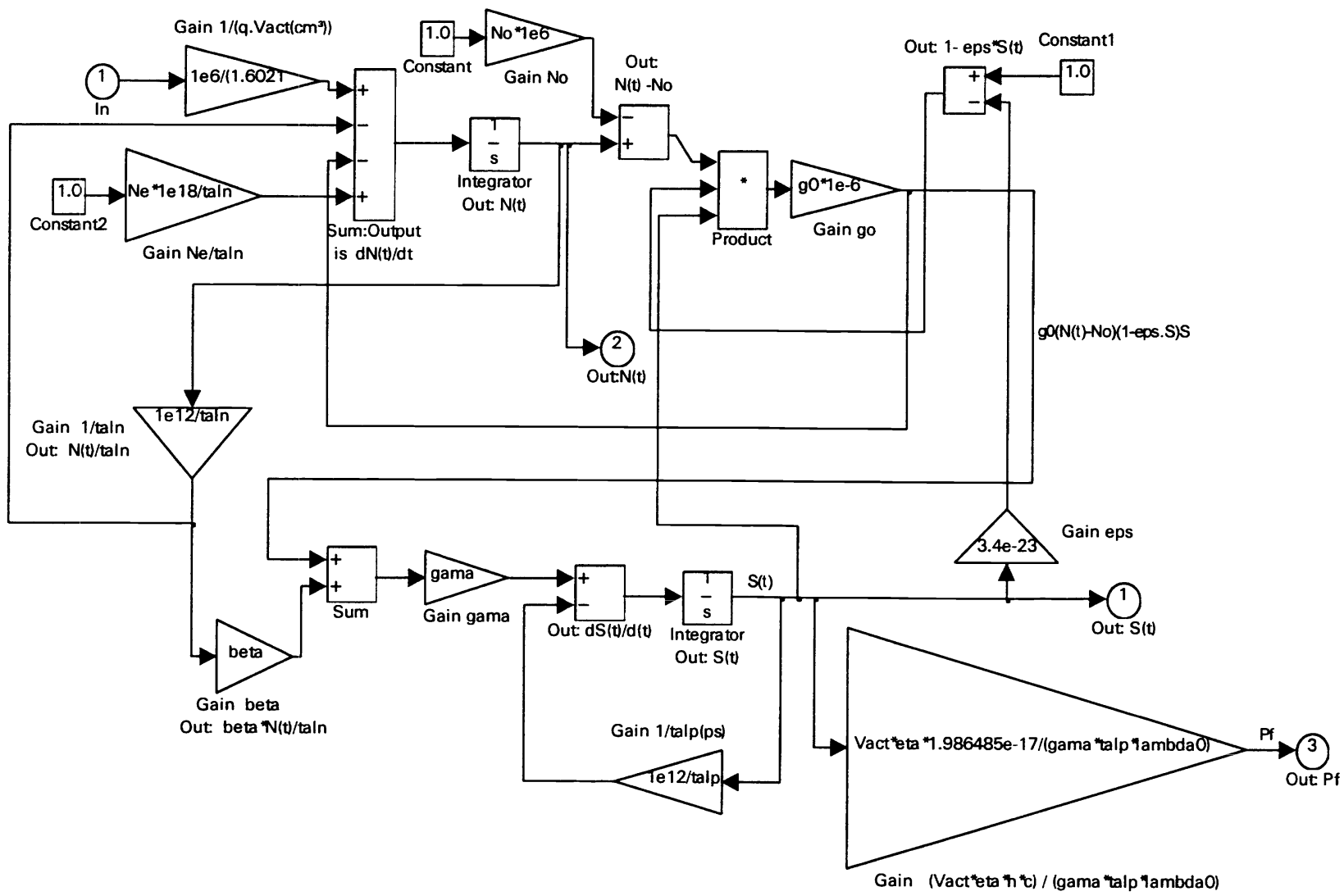

Fig 15 - Block diagram of "rate equations" module in time domain.

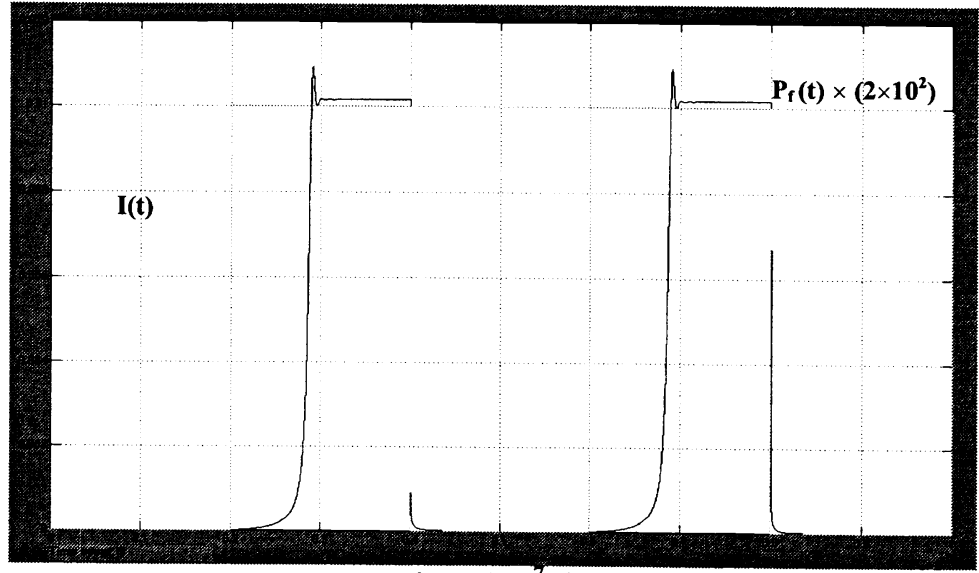

time $\left(10^{-7} \mathrm{~s}\right)$

Fig 16- Optical Output Power to a input current varying between 0 and $10 \mathrm{~mA}$ 


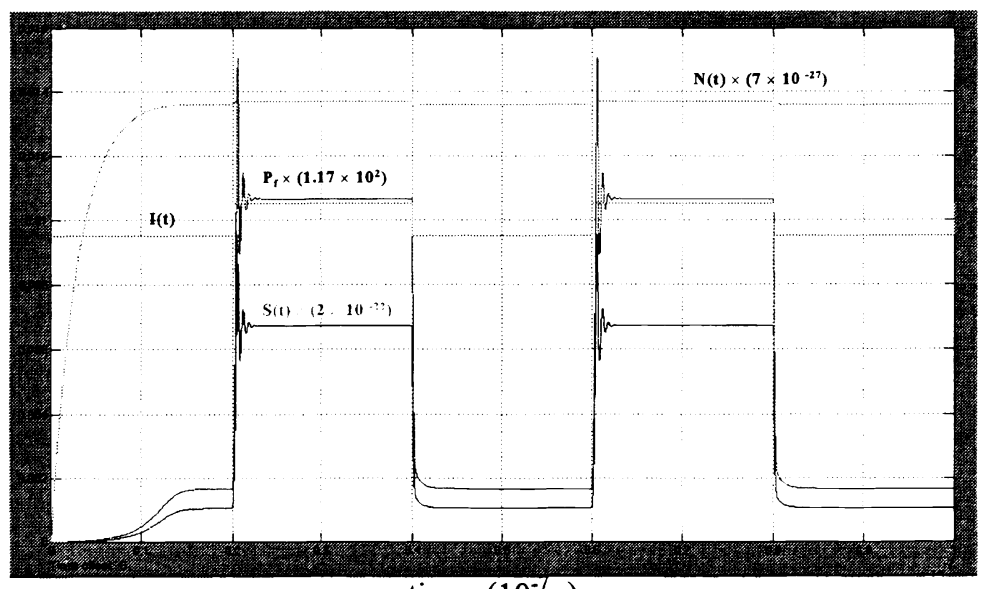

time $\left(10^{-7} \mathrm{~s}\right)$

Fig. 17- Variation of the photons density $S$, Carriers density $N$ and Optical output Power to an input current varying between 9.5 e 10.5 $\mathrm{mA}$

\section{TOOLBOX UTILIZATION EXAMPLE}

The blocks shown can be used to simulate a variety of optical links configurations, considering that the blocks disposition depends of the user to compose the system. In this example, it will be used a simple point-to-point topology, with purely didactic objectives. The simulated link can be seen in fig. 18, mounted exclusively with blocks contained in the five groups mentioned before.

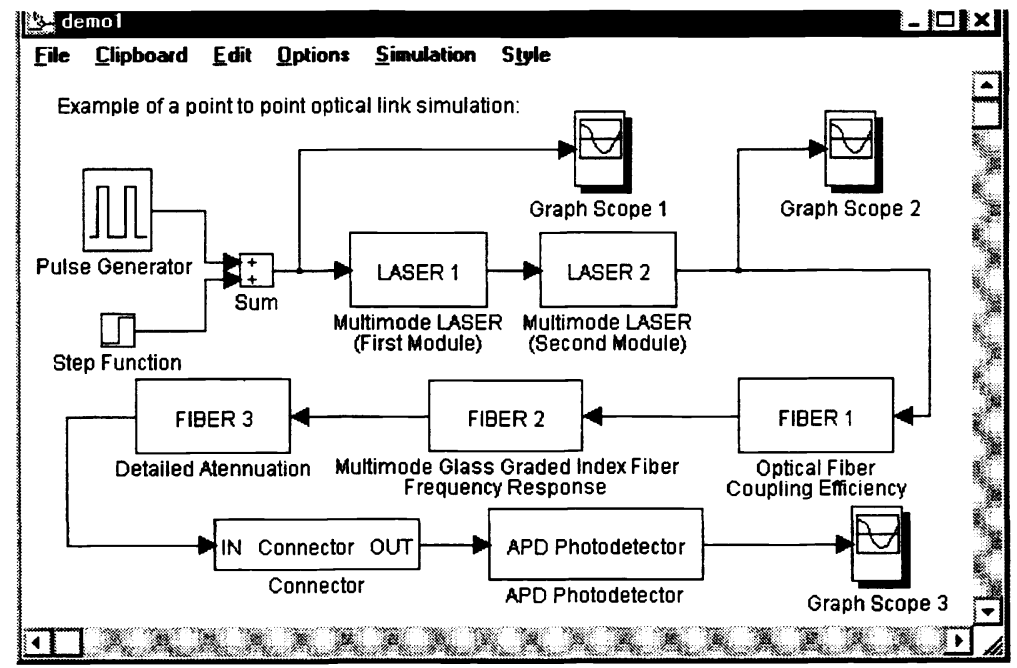

Fig. 18 - Simulation diagram for a point to point optical link.

Once the value definition for the blocks is done, the simulation can be started using the "Start" option in the "Simulation" menu. The result will soon appear on the screen, and is shown in Fig.19, in the time period of 0 to 10 ns. It must be noted that there are three different points of observation (graph scopes in Fig.18), all with the same magnitude scale. 


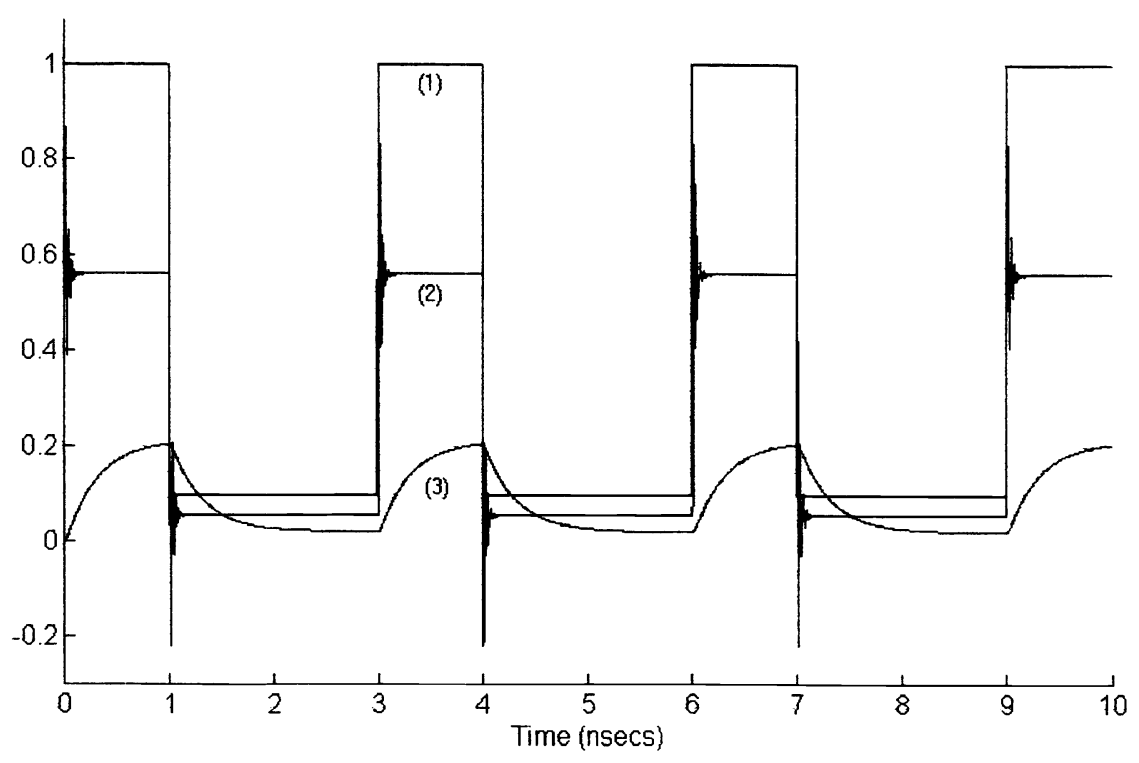

Fig. 19 - Simulation results for the diagram shown in Fig. 18.

\section{CONCLUSIONS}

The Simulink ${ }^{\mathbf{T M}}$ blocks described here have been used as a teaching tool for undergraduating students in basic courses of optical fiber communications at UFPa. The facilities of the computer simulation allows a better understanding of the theory, improving the student's yield, and the flexibility of the software permits the investigation of the influence of various parameters, proving to be a good didactic complement. Currently, the authors are developing models for filters, optical and semiconductor amplifiers, non linear fiber optics blocks to analyze high bit rate optical systems.

\section{REFERENCES}

1. Delyser, R. R., “ Using Mathcad in Eletromagnetic Education”, IEEE Trans. on Educ. ., Vol 39, n² 2, pp. 198-209, May 1996.

2. Iskander, M.F., “Computer -based Eletromagnetic education", IEEE Trans. on Microwave Theory Tech., vol 41, no 6/7, pp. 920-93, June/July 1993.

3. Mathworks, inc. "Simulink: A Program for Simulating Dynamic Systems- User's Guide." Natick, Massachusetts, 1995.

4. Lobão, P.MS, A Optical Fiber Communication Systems Simulator Software - MsC. Thesis - FEE/UNICAMP, 1992 (in portuguese).

5. Mena, Pablo V., "Rate Equation Based Models with a Single Solution Regime”, J. Ligthwave technol. Vol 15, no 4, p.p. 717 - 729, April 1997. 